\title{
Philosophiques
}

\section{L’énigme démocratique}

\section{Philip Pettit}

Volume 40, numéro 2, automne 2013

Autorité démocratique et contestation

URI : https://id.erudit.org/iderudit/1023701ar

DOI : https://doi.org/10.7202/1023701ar

Aller au sommaire du numéro

Éditeur(s)

Société de philosophie du Québec

ISSN

0316-2923 (imprimé)

1492-1391 (numérique)

Découvrir la revue

\section{Citer cet article}

Pettit, P. (2013). L'énigme démocratique. Philosophiques, 40(2), 351-368.

https://doi.org/10.7202/1023701ar

\section{Résumé de l'article}

La démocratie signifie d'abord et avant toute chose l'idée d'un contrôle populaire, et ce par l'ensemble des moyens possibles. Ces moyens donnent lieu à la légitimité. Mais ces contrôles populaires, du moins tels qu'ils sont entendus dans de nombreuses discussions, ne donnent pas lieu à la légitimité espérée. Les théories de la démocratie ne partagent pas une même conception des choses à ce sujet, ce qui donne lieu à une pluralité d'approches. Dans cet article, l'auteur soutient que la réponse la plus intéressante à cette question implique une réinterprétation de la notion de contrôle populaire.
Ce document est protégé par la loi sur le droit d'auteur. L'utilisation des services d’Érudit (y compris la reproduction) est assujettie à sa politique d'utilisation que vous pouvez consulter en ligne.

https://apropos.erudit.org/fr/usagers/politique-dutilisation/ 


\title{
L'énigme démocratique
}

\author{
PHILIP PETTIT \\ Laurance S. Rockefeller University Professor of Politics and Human Values at \\ Princeton University \\ ppettit@princeton.edu
}

\begin{abstract}
RÉSUMÉ. - La démocratie signifie d'abord et avant toute chose l'idée d'un contrôle populaire, et ce par l'ensemble des moyens possibles. Ces moyens donnent lieu à la légitimité. Mais ces contrôles populaires, du moins tels qu'ils sont entendus dans de nombreuses discussions, ne donnent pas lieu à la légitimité espérée. Les théories de la démocratie ne partagent pas une même conception des choses à ce sujet, ce qui donne lieu à une pluralité d'approches. Dans cet article, l'auteur soutient que la réponse la plus intéressante à cette question implique une réinterprétation de la notion de contrôle populaire.
\end{abstract}

ABSTRACT. - Democracy means popular control, by almost all accounts. And by almost all accounts democracy entails legitimacy. But popular control, at least as that is understood in many discussions, does not entail legitimacy. So something has got to give. Democratic theories divide on what this is, so that the question prompts a taxonomy of approaches. The most appealing answer, so the paper suggests, involves a reinterpretation of the notion of popular control.

Il y a trois affirmations plausibles que l'on fait communément au sujet de la démocratie, et qu'il paraitrait problématique de rejeter: elles constituent, pour ainsi dire, les axiomes de la discussion lorsque l'on s'intéresse à l'essence de la démocratie. Cependant, ces affirmations sont incompatibles les unes avec les autres, et cela constitue une énigme pour la théorie de la démocratie. Cet article présente et justifie chacune de ces affirmations, et montre que les approches classiques de la démocratie ne résolvent pas cette énigme de manière satisfaisante parce qu'elles n'y apportent qu'une solution ad hoc et ne parviennent à rétablir la cohérence de l'ensemble qu'en rejetant tout simplement l'un ou l'autre des axiomes en question. Nous esquisserons alors une stratégie de résolution différente, qui impliquera de réinterpréter et non de rejeter l'une des affirmations. Cela permettra de proposer une nouvelle manière de conceptualiser la démocratie en termes de régime de régulation délibérative. Dans une telle conception, les trois affirmations constituent un ensemble cohérent, tout en demeurant, chacune prise individuellement, parfaitement plausibles.

\section{L'énigme}

Les trois affirmations qui constituent l'énigme sont les suivantes:

- L'axiome de l'équivalence: "démocratie» veut dire le pouvoir au peuple.

- L’axiome de la légitimité: la démocratie implique la légitimité. 
- L'axiome de la tyrannie: le pouvoir au peuple n'implique pas la légitimité.

Il est clair que ces affirmations sont incohérentes les unes par rapport aux autres. Deux concepts, ceux de démocratie et de pouvoir au peuple, apparaissent comme logiquement équivalents: c'est là ce qu'il faut comprendre par "veut dire». Cependant, l'un de ces deux concepts, celui de démocratie, est censé impliquer celui de légitimité, alors que le second, celui de pouvoir au peuple, n'est pas censé le faire. Alors de deux choses l'une: ou bien on rejette l'une des affirmations, ou bien on réinterprète ces affirmations de manière à rétablir leur cohérence. Or aucune de ces affirmations ne paraît en soi ni ambiguë ni contestable. Aussi le fait qu'elles soient incohérentes les unes par rapport aux autres a-t-il quelque chose d'énigmatique.

Le premier axiome repose la signification du terme "démocratie», telle qu'on peut la déduire de son étymologie même. «Démocratie» vient de deux mots grecs, "démos » signifiant le peuple, et «kratos » le pouvoir, d'où l'idée que dans toute démocratie le peuple exerce une certaine influence, du moins quand l'extension de ce terme de "peuple» est donnée et ne pose pas de problème. [2] En particulier, il exerce une influence sur l'exercice du gouvernement ou par son intermédiaire. L'influence qu'il exerce ne doit cependant être ni partiale ni erratique, comme peut l'être, par exemple, celle du temps qu'il fait sur le gouvernement. Elle doit avoir un poids significatif, et viser systématiquement une fin qui soit considérée également par tous comme un bien - sans quoi elle n'offrira pas au peuple le type de pouvoir ou de contrôle requis. Cette première affirmation, ou axiome de l'équivalence, identifie la démocratie à un régime dans lequel le peuple dispose d'une influence, c'est-à-dire d'un pouvoir de ce type.

Le second axiome exprime l'intuition qui se trouve au fondement d'un argument classique dans le débat démocratique. J'utiliserais cet argument si j'affirmais, en vue de défendre un tant soit peu une loi ou une politique, que, ayant été introduites par l'intermédiaire de moyens démocratiques, celles-ci bénéficient donc de la garantie que leur confère un régime légitime, et on doit alors le reconnaître. Il est difficile de résister à cet argument, et c'est pourquoi la seconde affirmation nous paraît spontanément correcte. Bien entendu, elle n'offre pas grand-chose pour la défense d'une loi ou d'une politique donnée, puisque lorsque vous acceptez de reconnaître le régime comme légitime vous n'acceptez pas nécessairement l'idée que ses lois et ses politiques sont toutes invariablement justes, ou les meilleures. Il vous faudra surtout reconnaître que, puisqu'elles ont été adoptées au sein de ce régime - et, peut-on penser, en accord avec les procédures fixées par celui-ci - le gouvernement a le droit de promulguer et de faire appliquer ces lois et ces 
politiques, et il vous faudra accepter le fait que, si vous voulez vous y opposer, c'est au sein du système qu'il faudra le faire ${ }^{1}$.

S'opposer à une décision au sein du système signifie alors exploiter les ressources de la démocratie pour la remettre en cause dans les tribunaux, les lieux de débats politiques, les médias, ou même dans la rue. À la limite, cela peut même vouloir dire avoir recours à la désobéissance civile. Une telle désobéissance demeure dans les limites du système dans la mesure où vous acceptez le droit qu'a le gouvernement de vous punir pour avoir désobéi. En affichant une telle acceptation du régime - et par voie de conséquence, l'acceptation de la censure et de la punition - , vous soulignez l'intensité de votre sentiment et, par conséquent, la force de votre revendication à bénéficier du droit démocratique d'être entendu².

Je décris le troisième axiome comme étant celui de la tyrannie, parce qu'il recouvre l'affirmation commune selon laquelle le pouvoir du peuple peut donner lieu à une forme de tyrannie de la majorité. Cette affirmation est aussi vieille que la démocratie elle-même et souligne le fait qu'une majorité peut se servir du gouvernement en faveur de ses propres intérêts, et agir d'une manière qui revient à traiter les membres d'une minorité ou d'une autre comme n'étant pas égaux aux autres. Le mot de «tyrannie» est important, car il indique clairement qu'un système fondé sur l'idée que le pouvoir revient au peuple risque d'être non seulement imparfait ou injuste, mais tout à fait illégitime. L'idée qui se trouve derrière cette troisième affirmation est que le fait d'attribuer le pouvoir au peuple n'implique pas qu'il soit légitime, précisément parce que certaines décisions de la majorité peuvent se prendre systématiquement au détriment d'une minorité, de sorte qu'elles deviennent illégitimes.

\section{Les réponses classiques}

À l'énigme démocratique, on peut apporter trois réponses classiques. Plus exactement, en théorie démocratique, il y a trois positions classiques qui

1. Remarquez que je définis ici la légitimité d'un régime sans la relier à une obligation, pro tanto ou autre, de respecter les prescriptions du régime. Je pense tout simplement qu'une telle légitimité a plutôt un lien avec l'obligation, dans le cas où vous vous opposeriez aux lois, de vous y opposer au sein du système. Voir Pettit, 20I 2, et, sur des sujets connexes, Simmons, I979.

2. Il peut également être encore mieux de s'opposer à un régime illégitime au sein du système, bien entendu, en particulier par la désobéissance civile. Dans ce cas, la désobéissance civile signifierait traiter le régime comme s'il était légitime, tout en pensant qu'il ne l'est pas en réalité. Une telle tactique pourrait contribuer à installer des moyens de pression qui pousseront le régime à adopter un modèle d'organisation et d'action de plus en plus légitime. Tenir un individu ou une institution pour responsables lorsqu'ils ne peuvent pas être adéquatement tenus pour tels peut contribuer à les rendre responsables. Cela peut les «responsabiliser» (Garland, 200I; Pettit, 2007). De la même manière, traiter un régime comme légitime, au moyen de la désobéissance civile disons, peut permettre d'obtenir l'appui d'autrui et d'exposer le gouvernement à la publicité de manière à ce que le système fonctionne de manière toujours plus légitime. 
proposent des réponses différentes à cette énigme, chacune réfutant un axiome différent. Pour des raisons qui s'éclairciront au fil de la discussion, je les nomme populisme, libéralisme et éclectisme.

La réponse populiste accepte l'idée que la démocratie signifie le pouvoir au peuple, et qu'elle implique également la légitimité; mais elle évite le problème en niant la troisième affirmation - l'axiome de la tyrannie - et part du principe que, si un système repose sur le pouvoir populaire, c'est un régime légitime. L'idée est de s'en tenir à la logique de la loi de la majorité, et d'accepter la conclusion selon laquelle cette loi est toujours une loi légitime, même si, d'une manière ou d'une autre, elle s'exerce au détriment d'une minorité. Cette position est populiste dans la mesure où elle donne la priorité aux droits collectifs du peuple sur les droits individuels de ses membres, notamment ceux qui se trouvent dans le camp des minorités.

La réponse libérale accepte l'idée que la démocratie signifie le pouvoir au peuple, comme l'affirme le premier axiome, et que le pouvoir au peuple n'implique pas la légitimité, comme l'affirme le troisième; elle évite le problème en rejetant la seconde affirmation selon laquelle la démocratie implique la légitimité. Je tiens cette réponse pour libérale, car on l'associe en particulier à l'argumentation développée par William Ricker (I982) en faveur du libéralisme et contre le populisme. Il y accepte l'identification entre démocratie et pouvoir au peuple, mais soutient que, tel qu'il est exemplifié dans les systèmes de vote habituels, le pouvoir populaire ne garantit pas la légitimité: il est tout à fait compatible avec une violation des droits des minorités - ce qui, intuitivement, constitue l'illégitimité d'un régime. Il accepte ainsi la conclusion selon laquelle la démocratie n'implique pas la légitimité. Pour qu'un régime soit reconnu comme légitime, propose-t-il, la prise de décision politique ne devra pas seulement émaner d'une source démocratique, mais aussi respecter les droits individuels de ceux qu'elle affecte. Selon cette approche, la démocratie n'est pas garante de la légitimité. Il faut, pour qu'un système soit légitime, que cette prise de décision soit contrebalancée par un régime contre-majoritaire de droits individuels. Ce régime structurera son action autour d'une constitution qui limitera l'étendue du champ d'application de la volonté démocratique.

Alors que le populisme rejette la troisième affirmation et le libéralisme la seconde, l'éclectisme rejette la première. Il affirme qu'on doit identifier la démocratie non pas avec le pouvoir au peuple, mais seulement avec une répartition éclectique du pouvoir populaire, sous la contrainte d'un facteur indépendant: d'ordinaire un certain ensemble de droits individuels. L'éclectisme embrasse une conception dite "composée" de la démocratie (par exemple Mayerfield, 2009). Pour être considéré comme démocratique, un régime devra ainsi contenir à la fois l'élément de pouvoir et l'élément de contrainte. À lui seul, l'élément de pouvoir n'assurera pas la démocratie. Cette précision ayant été apportée, le deuxième et le troisième axiomes peuvent être conservés: la démocratie au sens éclectique impliquera la 
démocratie, ce qui ne serait pas le cas du pouvoir populaire à lui seul. On adopte souvent ce type de position dans la théorie du droit et la théorie politique contemporaines. On la trouve dans la conception de la démocratie défendue par Ronald Dworkin (2006, I3 I) lorsqu'il écrit: "Une communauté qui néglige constamment les intérêts d'une minorité ou d'un groupe n'est, pour cette raison-même, pas démocratique.»

La stratégie utilisée par les trois positions que nous venons de décrire est parfaitement claire. Chacune endosse deux des axiomes et dérive la fausseté du troisième de l'acceptation des deux premiers. L'éclectisme soutient que la démocratie, mais pas le pouvoir populaire, implique la légitimité, et en conclut que l'on ne peut tenir la démocratie et le pouvoir au peuple pour équivalents, la première requérant un régime de droits individuels. Le libéralisme soutient que la démocratie équivaut à donner le pouvoir au peuple, mais que le pouvoir populaire n'implique pas la légitimité; il conclut que la démocratie n'implique pas non plus la légitimité: il faut là aussi que certains droits soient respectés. Le populisme soutient que la démocratie équivaut au pouvoir du peuple, et qu'elle implique donc la légitimité. Il conclut ainsi que le pouvoir populaire implique la légitimité. On peut représenter cet ensemble sous la forme du tableau suivant:

\begin{tabular}{|l|l|l|l|}
\hline & $\begin{array}{l}\text { Axiome de } \\
\text { l'équivalence }\end{array}$ & $\begin{array}{l}\text { Axiome } \\
\text { de la légitimité }\end{array}$ & $\begin{array}{l}\text { Axiome } \\
\text { de la tyrannie }\end{array}$ \\
\hline Éclectisme & Non & Oui & Oui \\
\hline Libéralisme & Oui & Non & Oui \\
\hline Populisme & Oui & Oui & Non \\
\hline
\end{tabular}

L'une de ces trois positions a-t-elle de quoi nous séduire? Je ne le pense pas. Chacune d'entre elles souffre d'un défaut manifeste, à savoir que dans chaque cas la raison supposée de rejeter l'axiome visé n'est rien d'autre que l'idée selon laquelle, ce faisant, on résoudra l'énigme. En ce sens, le rejet est ad hoc. L'éclectisme est séduisant dans la mesure où il préserve les axiomes de la légitimité et de la tyrannie; le libéralisme parce qu'il préserve ceux de l'équivalence et de la tyrannie; le populisme enfin, parce qu'il préserve les axiomes de l'équivalence et de la légitimité.

Il n'y aurait pas là de problème particulier si, dans chaque cas, l'axiome rejeté n'était pas si convaincant. Mais, intuitivement au moins, chacun de ces axiomes paraît irréfutable. L'axiome de l'équivalence présente clairement l'idée, essentielle pour la démocratie, selon laquelle le peuple gouverne en exerçant le contrôle sur le gouvernement, ou par lui. Le rejeter sous prétexte que le peuple devrait gouverner sous la contrainte d'un ensemble donné et indépendant de droits semble revenir à redéfinir ce qu'est la démocratie selon ses propres fins, c'est-à-dire à manipuler les lois. Les choses 
seraient différentes si l'argument consistait à dire que la notion même de pouvoir populaire, dans le sens qui convient, requiert un régime de droits: c'est le type de position que je défendrai par la suite ${ }^{3}$. La position éclectique est que la démocratie n'est pas seulement une affaire de pouvoir populaire, en dépit de l'étymologie et de la signification de ce terme. Elle requiert l'intervention d'un autre élément, qui n'a rien à voir avec la notion de pouvoir ${ }^{4}$.

L'axiome de la légitimité présente une affirmation que l'on retrouve classiquement sur deux fronts dans la discussion sur l'essence de la démocratie. Elle apparaît, en premier lieu, dans l'affirmation commune selon laquelle si vous admettez qu'un régime est démocratique mais niez qu'il soit légitime, alors vous êtes un élitiste qui rejette les termes de l'échange démocratique. Vous dites qu'il ne suffit pas, pour que le régime soit légitime, que le peuple gouverne: il faut qu'il gouverne bien, c'est-à-dire qu'il gouverne à la lumière d'une autorité que l'on présume supérieure. L'axiome de la légitimité intervient aussi, en second lieu, dans l'affirmation commune que l'on retrouve dans la classification des différents régimes, et selon laquelle si un système de gouvernement paraît intuitivement illégitime, par exemple parce qu'il n'accorde pas d'indépendance à la justice, alors ce régime n'est pas démocratique à proprement parler. Si l'illégitimité implique l'absence de démocratie, alors il faut que la démocratie implique la légitimité.

L'axiome de la tyrannie s'impose intuitivement encore plus fortement que les deux autres axiomes. Cet axiome se retrouve au premier plan des commentaires canoniques que l'on peut trouver en ce qui concerne la pratique démocratique, tant à l'époque classique, ou moderne, que contemporaine. Il a toujours paru évident que le gouvernement du plus grand nombre pouvait avoir des conséquences délétères sur la vie de quelques-uns, et on a toujours considéré comme allant de soi qu'un abus de pouvoir systématique mené contre une minorité constituait quelque chose d'illégitime. Le contrôle électoral peut conduire à la tyrannie de la majorité, or la tyrannie est le paradigme même de ce qu'est un régime illégitime.

Les trois réponses que nous venons de rappeler, ainsi que les critiques que l'on peut y opposer, trouvent une interprétation naturelle dans les termes de la conception électoraliste ou représentative du peuple comme ensemble des membres de la société, considérés comme des sujets indépendants. Mais

3. Pour mémoire, les formules utilisées par Ronald Dworkin laissent parfois entendre que les droits individuels devraient être pris en considération, non à titre de contrainte indépendante pesant sur le contrôle populaire, mais à titre de contrainte requise par la notion de pouvoir populaire elle-même.

4. Stephen Holmes (I995, 2006), endossant quelque chose qui ressemble à la position éclectique, l'adoucit en affirmant que, si la notion de démocratie ne requiert pas un régime de droits individuels, l'organisation de la démocratie, en revanche, l'exige. L'idée est qu'un régime démocratique serait écrasé par le poids des prises de décision si les droits individuels ne résolvaient pas un grand nombre de questions. Voilà qui est probablement la meilleure défense que l'on puisse présenter en faveur de ce qui demeure une perspective typiquement éclectique. 
il vaut la peine de remarquer qu'elles se retrouvent aussi dans la conception rivale, participative, du peuple en tant qu'il constitue une assemblée dont les membres ont le devoir d'agir sous le signe de l'unité. Il s'agit là du peuple considéré en bloc plutôt qu'en masse, selon l'image que Hobbes (I994) et Rousseau (I973) ont donnée du peuple souverain. Hobbes pensait que le peuple pouvait légitimement adopter ce statut d'auto-incorporation et de souverain, même s'il ne pensait pas que cela fût nécessaire pour que le gouvernement soit légitime. En réalité, il préférait l'accord en vertu duquel le peuple serait représenté par un souverain individuel. Par contraste, comme on sait, Rousseau soutenait que le peuple, en tant qu'assemblée, constituait le seul souverain légitime.

Interprété dans les termes du modèle électoraliste, l'éclectisme affirme qu'il ne suffit pas que le peuple dispose d'un contrôle électoral pour que l'on ait affaire à une démocratie; le libéralisme affirme quant à lui que, si ce contrôle est suffisant pour la démocratie, la légitimité n'est pas garantie pour autant, et le populisme considère que le contrôle électoral ne permet pas de rendre compte du caractère illégitime d'une tyrannie de la majorité. Mais toutes ces affirmations trouvent aussi leur traduction dans le modèle de l'assemblée. L'éclectisme dirait qu'il ne suffit pas que le peuple gouverne en assemblée pour qu'on ait affaire à une démocratie; selon le libéralisme, le fait qu'une assemblée gouverne suffit pour la démocratie, mais ne garantit pas la légitimité; et le populisme, de la manière la plus effrontée qui soit, dirait que le contrôle par une assemblée n'autorise pas la tyrannie illégitime de la majorité.

\section{Identification de la stratégie de réinterprétation}

Chacune des trois positions que l'on vient d'identifier peut être soutenue, mais au regard des problèmes qu'elles posent aucune ne s'avère particulièrement attractive. L'éclectisme demande une redéfinition radicale de ce qu'est la démocratie, le libéralisme un rejet de l'idée fondamentale que l'approbation démocratique est une garantie de légitimité, et le populisme, l'acceptation de l'idée très discutable selon laquelle aucune contrainte collective ne peut peser de manière illégitime sur la vie des individus. Vers quoi devonsnous alors nous tourner?

Les stratégies que nous avons discutées impliquent toutes le rejet d'une des affirmations, par conséquent il nous faut payer le prix de son abandon. Une autre stratégie, si l'on veut résoudre l'énigme, consisterait à réinterpréter plutôt qu'à rejeter. Elle trouverait des raisons d'affirmer, si on les comprenait bien, que chacune de ces affirmations prises individuellement est vraie, mais que prises toutes ensembles elles se contredisent. Y a-t-il de l'espoir de ce côté-là ?

Si la stratégie de la réinterprétation devait fonctionner, au moins l'un des termes essentiels que sont ceux de "démocratie", de "pouvoir au peuple» et de «légitimité» devrait avoir un sens différent dans les deux 
axiomes où il apparaît. Or les termes de "démocratie» et de "légitimité » sont censés être utilisés dans un sens approprié, quelle que soit la manière dont on les interprète. L'idée est que, peu importe la manière dont on comprend la démocratie, celle-ci implique le pouvoir au peuple et conduit à la légitimité, et, peu importe la manière dont on comprend la légitimité, celle-ci est le résultat de la démocratie, mais pas du pouvoir populaire. Par conséquent, le seul espoir pour une stratégie de réinterprétation est qu'elle soit capable d'établir que le "pouvoir au peuple» prend un sens particulier dans l'axiome de l'équivalence, et un autre dans l'axiome de la tyrannie.

Dans l'axiome de la tyrannie, on associe clairement le pouvoir au peuple à la loi du plus grand nombre - il s'agit, en gros, de la règle de la majorité. Si on le prend en ce sens, cet axiome est clairement vrai. Autrement dit, la stratégie réinterprétative ne fonctionnera que si on peut réinterpréter l'idée du pouvoir populaire, dans l'affirmation de l'équivalence, en un sens non majoritarianiste - plus précisément, de manière à préserver l'idée que la démocratie entraîne la légitimité. Si on comprend ainsi le pouvoir populaire en un sens dans l'axiome de l'équivalence, et en un autre dans l'axiome de la tyrannie, alors il n'y a plus de contradiction problématique entre les trois affirmations.

Nous avons vu tout à l'heure que si le pouvoir est exercé démocratiquement par le peuple, il faut que le peuple exerce un degré significatif d'influence sur le gouvernement, et cette influence doit toujours viser une fin jugée désirable et partagée. L'exercice majoritaire de l'influence peut sans doute être significatif, que ce soit dans l'électorat ou dans une assemblée, mais la raison pour laquelle il pose un problème est qu'il semble bien pouvoir toujours viser une fin majoritaire, et non pas partagée: la satisfaction du plus grand nombre, qui est relativement indifférent à celle des moins nombreux. Cette fin peut être systématiquement poursuivie parce que les votants agissent comme autant de factions en faveur des fins de leur groupe, et que la fin de la faction majoritaire l'emporte, ou bien parce que chacun des votants agit en tant qu'individu en faveur de ses propres fins, et que la fin qui est poursuivie par le plus grand nombre — c'est-à-dire par la faction majoritaire - l'emporte. Mais si l'axiome de la tyrannie interprète le contrôle populaire comme une influence majoritaire en faveur des fins de la majorité, alors nous pouvons peut-être comprendre la notion de pouvoir, dans l'affirmation de l'équivalence, comme une forme d'influence qui diffère de tous les autres processus majoritaires.

Si cela est plausible, nous pouvons alors proposer une version crédible de la stratégie réinterprétative. Nous pourrons reformuler les trois axiomes sous une forme qui les rendra individuellement convaincants, mais néanmoins collectivement cohérents. Cette reformulation pourra ressembler à ce qui suit:

- Axiome de l'équivalence: la démocratie signifie le pouvoir au peuple, non majoritaire. 
- Axiome de la légitimité: la démocratie implique la légitimité.

- Axiome de la tyrannie: le pouvoir au peuple n'implique pas par luimême la légitimité.

Y a-t-il une interprétation plausible du pouvoir populaire, et non majoritaire, qui permette de concevoir ce pouvoir comme répondant bien à l'idée de démocratie? Le défi est ici de trouver une conception non majoritarianiste des rapports entre moyens et fins, laquelle nous permettrait de dire que le peuple exerce le pouvoir populaire associé à la démocratie quand il utilise ce moyen en vue de cette fin. Plus spécifiquement, le défi est de trouver une conception de ces rapports que nous puissions espérer intégrer à une représentation adéquate des institutions: il ne doit pas s'agir de quelque chose que les institutions réelles seraient incapables de promouvoir de manière fiable.

Une fois la question posée de cette manière, il devient clair qu'il y a en lice au moins une réponse possible dans la littérature. On la trouve dans le modèle de l'assemblée qui fut présenté par Rousseau dans Le contrat social, publié en I762. Selon Rousseau, à proprement parler la démocratie exige non seulement que le peuple légifère en assemblée, comme le montre son modèle de l'assemblée, mais qu'il agisse également en tant qu'exécuteur de ses propres lois - ce qui, à ses yeux, n'est pas désirable. Cependant, abstraction faite de la terminologie propre à Rousseau, on peut raisonnablement traiter le gouvernement par l'assemblée, tel qu'il le propose, comme un modèle de démocratie 5 .

Rousseau accepte le second axiome, selon lequel la démocratie, prise dans le sens approprié d'assemblée du peuple, implique la légitimité, et il approuve aussi, conformément à la tradition républicaine, le troisième axiome selon lequel le pouvoir populaire en lui-même - le contrôle de la majorité, de l'assemblée - n'implique pas la légitimité. Pour résoudre l'énigme, il réinterprète le premier axiome, l'affirmation de l'équivalence selon laquelle la démocratie équivaut au contrôle populaire, au contrôle de l'assemblée. Selon lui cette affirmation est vraie pour autant que le pouvoir populaire - ou contrôle de l'assemblée - est contraint, de sorte qu'il est exercé par l'assemblée prise comme un tout, dans le cadre de la poursuite des volontés de l'assemblée prise comme un tout: la volonté générale. Ainsi contraintes, les décisions de l'assemblée seront immunisées contre les intérêts des corporations, des factions ou des coalitions. Rousseau peut ainsi

5. Hobbes (I994), bien entendu, opte pour une voie différente. Il affirme que le troisième axiome devrait être rejeté, puisque selon lui il n'y a pas de tyrannie. Tout individu ou corps qui exerce la souveraineté gouverne effectivement, préservant ainsi de tout retour à l'état de nature, et il est un gouvernant légitime. Cette position dérive d'une théorie beaucoup plus large de la nature de l'État ou du bien commun. Hobbes considère que la démocratie pourrait donner lieu à la légitimité, sans que l'on se retrouve confronté à l'énigme de la démocratie, mais il pense également que d'autres régimes efficaces peuvent être tout aussi légitimes. 
représenter l'axiome de l'équivalence comme étant cohérent avec l'affirmation selon laquelle le contrôle de l'assemblée, puisqu'il peut aussi n'être soumis à aucune contrainte, n'implique pas automatiquement la légitimité. Il reformule les trois axiomes sous la forme cohérente suivante:

- Axiome de l'équivalence: la démocratie signifie le pouvoir, adéquatement contraint, à l'assemblée.

- Axiome de la légitimité: la démocratie implique la légitimité.

- Axiome de la tyrannie: le pouvoir de l'assemblée n'implique pas en lui-même la légitimité.

La solution de Rousseau n'est cependant pas d'un grand intérêt par elle-même, pour au moins trois raisons. Tout d'abord elle présuppose, de manière tout à fait irréaliste, qu'une assemblée regroupant tous les membres d'une société pourrait s'acquitter de la tâche de gouverner. Il n'y a aucune version plausible de cette idée qui puisse s'appliquer, pas plus à une démocratie électorale qu'à une démocratie d'assemblée. Ensuite, la volonté générale que la prise de décision démocratique est censée incarner demeure quelque chose de bien obscur ${ }^{6}$. Et enfin, les mesures proposées en vue de contraindre l'assemblée et qui permettent l'incarnation de la volonté générale sont loin d'être vraiment convaincantes. On pourrait proposer que l'assemblée ne puisse voter qu'à propos des affaires générales, et non à propos de questions qui présentent un intérêt particulier pour certains individus; on pourrait aussi proposer d'éviter toute discussion qui risquerait de conduire à la formation de factions. Mais il est loin d'être clair que chacune de ces mesures puisse être mise en œuvre de manière fiable.

L'idée essentielle de l'approche rousseauiste est que le pouvoir exercé par le peuple devrait être contraint de manière à ce qu'il reflète sa volonté

6. En fait, on ne peut pas identifier la préférence ou la volonté d'un groupe d'agents la volonté collective - à celle en faveur de laquelle une majorité se prononce. Prenons les simples préférences ponctuelles concernant la question de savoir si $p$, si $q$, et si $p \& q$, parmi un groupe de trois individus A, B et C. Ce scénario est une déclinaison du scénario sur les jugements qui est discuté un peu plus loin dans l'article. A et $C$ pourraient préférer que $p$, de sorte qu'une majorité se prononce en faveur de cela, B seul étant contre. B et C pourraient préférer que $q$, de sorte qu'une majorité est en sa faveur, A seul étant contre. Mais A et B préférerons de manière cohérente que non- $p \& q$, de sorte qu'une majorité préfère que $p$, une majorité préfère que $q$, et une majorité préfère que non- $p \& q$. La seule résolution que peut prendre le groupe, pour agir comme un agent collectif, consiste à prendre parti contre la préférence de la majorité sur certains points: en l'occurrence, en tant que groupe, il formera la volonté que $p$, que $q$, et aussi, contre la majorité, que non- $p \& q$. Voir Pettit, 200I, chap. 5 ; List and Pettit, 2002; Pettit, 2003 b; List, 2006; List and Pettit, 20II. Rousseau pourrait accepter cette idée, pour autant qu'il montre que la volonté collective n'est pas nécessairement identique à la volonté de la majorité. Mais, bien entendu, cela montre également, contre ce qu'affirme Rousseau, que la volonté collective n'est pas nécessairement identique à celle de la majorité, même quand cette volonté est formée en respectant les contraintes appropriées. Et elle ne montre nullement que la volonté collective ainsi formée sera préservée de toute influence partisane. 
générale, notamment parce que celle-ci n'est pas toujours bien reflétée par le vote de la majorité. Selon lui, les moyens par lesquels s'exerce le pouvoir populaire devraient être limités de manière à ce que l'incarnation de cette volonté soit prise pour but, afin d'éviter une tyrannie de la majorité et de mettre en place un régime assurant la légitimité. Étant donné que la volonté générale rousseauiste ne semble pas constituer un objectif atteignable vers lequel s'exercerait le contrôle populaire, y a-t-il cependant une option différente qui puisse jouer ce rôle? Quelles sont les autres fins populaires, et les moyens populaires afférents, que l'on pourrait identifier comme étant essentiels pour le type de pouvoir populaire que le premier axiome identifie à la démocratie?

\section{La stratégie réinterprétative en application}

Si l'on renonce à faire de la volonté générale le but du pouvoir populaire, la solution qui se présente le plus immédiatement à l'esprit est celle que suggère l'opposition entre volonté et raison. Quand il s'agit de réfléchir au pouvoir dont un sujet individuel dispose sur sa vie, l'une des grandes oppositions à laquelle on se trouve confronté est celle qui oppose l'école de pensée volontariste, associant ce pouvoir au pouvoir de la volonté - peu importe la manière dont on l'entend - à l'approche intellectualiste qui l'identifierait plutôt au pouvoir de la raison. Cela laisse alors entendre que, si nous devions trouver une solution à l'approche rousseauiste qui permette d'éviter l'énigme démocratique sur un mode parallèle, nous devrions réfléchir à la possibilité d'une forme de pouvoir populaire qui soit conçu, tant sur le plan des fins que sur le plan des moyens adoptés, de manière à incarner ce que l'on pourrait envisager comme la raison du peuple.

L'idée d'un système de pouvoir populaire fondé sur la raison se décline en deux variantes, selon que ce système est censé donner corps soit au raisonnement que mène le peuple à propos de ce que, collectivement, il devrait faire, soit aux raisons qu'il valide comme autant de considérations relatives à la question de ce que, collectivement, il devrait faire. On utilise en général le terme de "démocratie délibérative " pour désigner la première proposition, bien qu'on ne l'applique souvent à la démocratie que dans l'une ou l'autre de ses incarnations locales seulement: une réunion publique en ville, ou une association à but non lucratif, ou un comité officiel (Cohen, I989; Gutmann and Thompson, I996; Bohman and Rehg, I997; Elster, I998). La seconde proposition, que je trouve plus attrayante, se retrouve parfois à l'arrière-plan des discussions concernant la démocratie délibérative, mais il est rare qu'on la formule correctement. Tandis que la première défend l'idée d'une démocratie menée de manière délibérative, la seconde se prononce en faveur d'une démocratie régulée de manière délibérative. Elle met l'accent, non pas d'abord sur l'exercice du raisonnement par le peuple, mais sur le fait que le gouvernement - soit représentatif, soit participatif - doit rendre compte de ses décisions sur la base des raisons qui sont invoquées dans le 
débat public, et que celles-ci doivent être acceptées par le peuple de façon générale.

Ceux qui prônent la démocratie régulée de manière délibérative recommandent que le raisonnement s'effectue au sein des membres du groupe concerné, un raisonnement qui porte en particulier sur la question de savoir quelle décision servira au mieux le groupe, et non sur celle de savoir ce qui permettra de promouvoir les intérêts particuliers de ses membres (Coleman and Ferejohn, I986). Mais parce que dans la démocratie il y a toujours des désaccords, cette délibération individuelle devra aboutir, à un moment donné, à un vote. Et parce que le fait de voter sur des affaires reliées entre elles est toujours susceptible de donner naissance à un ensemble incohérent de jugements de la part du groupe, l'idéal de la démocratie délibérative exige des membres qu'ils soient préparés à délibérer en tant que groupe, et non pas seulement individuellement, sur ce qui est le plus à même de résoudre de telles incohérences. Une délibération collective de ce genre ne pourra cependant être conduite ailleurs qu'au sein d'un groupe de taille relativement réduite. Par conséquent, l'idéal d'une démocratie régulée de manière strictement délibérative est nécessairement impossible à mettre en œuvre à l'échelle nationale (Pettit, 2003a).

Le seul point qui manque peut-être de clarté dans cet argument est l'affirmation selon laquelle, quand elle se prononce sur des questions liées les unes aux autres, la majorité est susceptible de donner lieu à un ensemble de jugements contradictoires. Mais cela peut être démontré (Pettit, 200I, chap. V; List and Pettit, 2002; Pettit, 2003 b; List and Pettit, 20I I). Pour l'illustrer, prenons un petit groupe de seulement trois personnes, A, B et C, qui doivent voter, au même moment ou à des moments différents, pour savoir si $p$, si $q$ et si $p \& q$. A et $\mathrm{C}$ peuvent voter que $p, \mathrm{~B}$ que non- $p$. B et $\mathrm{C}$ peuvent voter que $q$, et $\mathrm{A}$ que non- $q$. Ainsi, à titre de majorité, $\mathrm{A}$ et $\mathrm{B}$ voteront de manière cohérente que non- $p \& q$. Car après tout, A pense que non- $q$ et $\mathrm{B}$, que non- $p$. S'il agit selon le principe strict du vote à la majorité, ce groupe devra soutenir que $p$, que $q$ et que non- $p \& q$. Or aucun groupe d'agents sensés ne sera satisfait par un tel ensemble de jugements incohérents, si du moins ces jugements sont étroitement liés à une prise de décision. Par conséquent, il faudra que le groupe délibère, en tant que groupe, sur la question de savoir lequel des jugements majoritaires il doit rejeter afin de s'assurer que ce groupe agisse selon une vision cohérente.

Mais si un pouvoir populaire basé sur la pratique de la délibération ne semble pas envisageable, qu'en est-il de l'idée voisine de pouvoir populaire basé sur une régulation délibérative, c'est-à-dire une régulation assurée par les raisons populaires? Pour introduire cette possibilité, il nous faut répondre à trois questions: qu'est-ce que les raisons populaires? Comment peuventelles réguler le gouvernement? Et un tel système de régulation démocratique nous permet-il de résoudre l'énigme démocratique? 


\section{Première question}

Supposons que, comme cela se produit souvent dans la démocratie électorale, les membres d'une société débattent constamment de la question de savoir ce que leur gouvernement, ou eux-mêmes, devraient faire en telle ou telle circonstance. Nous pouvons imaginer que les discussions ont lieu chez les gens, dans les couloirs de bureaux et les bars, aussi bien que dans des débats plus formels dans les médias, dans les réunions politiques, ou au sein du Parlement ou du Congrès. Dans une démocratie véritablement fonctionnelle, de telles discussions, normalement, ne finissent pas en bagarres, même si elles font apparaître des divisions entre les membres et peuvent mettre au jour des désaccords assez profonds. Que ce soit le cas signifie que les participants doivent se montrer capables d'identifier les considérations présentées par chacun quand il défend ses positions, et dont il peut s'attendre à ce que les autres les trouvent adéquates ou pertinentes pour les questions d'ordre collectif qui font l'objet du débat. Les désaccords viendront du fait que les gens donnent un poids différent à ces différentes considérations, ou qu'ils ont des opinions divergentes sur la réalité en cause — ou encore, bien entendu, du fait qu'il y a des problèmes de cohérence ou de logique d'un côté ou de l'autre. Mais quelle que soit la quantité de désaccords qui surgit de telles sources, le fait que les discussions entre les différentes parties se poursuivent, sans déboucher sur un conflit ouvert, signifie que ces parties doivent être capables de trouver ou de forger des raisons en faveur de ce qu'elles avancent, et qui seront jugées valables par tous comme autant de considérations à traiter dans le processus collectif de prise de décision. Ces raisons seront de toute manière pertinentes pour résoudre le problème de savoir ce que la collectivité doit faire; ce ne seront pas des raisons uniquement liées à des intérêts partisans, ou dépendant de croyances partagées seulement par certaines parties de la communauté, ou n'ayant aucun lien, d'une manière ou d'une autre, avec les problèmes communs.

Toutes les parties apprennent à trouver leur chemin dans les discussions concernant les décisions collectives, y compris les discussions qui dépassent leur cercle immédiat ou qui touchent différents cercles de la société: il est de leur devoir de se familiariser avec des considérations de cette nature. Ces considérations constitueront des raisons en faveur ou en défaveur de certaines décisions collectives, que tous reconnaîtront comme pertinentes, chacune bénéficiant incontestablement de cette reconnaissance, et ainsi de suite, selon l'ordre habituel. Elles constitueront une monnaie commune, en principe susceptible d'être constamment enrichie, pour l'évaluation et la régulation de la prise de décision collective.

À quoi peut-on s'attendre quant à la variabilité des raisons communes ou populaires qui peuvent émerger dans une société et servir à la régulation du gouvernement? Il faut que certaines de ces raisons soient reconnues comme la condition même pour qu'une monnaie commune de raisons populaires puisse émerger, se développer, et gagner un pouvoir d'influence. Cela 
comprend des considérations relatives au respect, lesquelles empêchent que des individus soient traités comme moins que des personnes, accessibles par la parole et le raisonnement - par exemple, prendre parti contre toute restriction du droit à la parole, et pour la limitation du champ d'action du gouvernement - , et d'autres considérations relatives à l'égalité, qui empêchent de traiter toute partie comme étant moins digne d'un égal respect. Ces considérations pourraient militer contre le fait de nier à certaines personnes un droit égal à la parole et à l'association, par exemple, ou de leur refuser une égale immunité face à certaines actions du gouvernement. D'autres considérations pourraient être plus ou moins universellement irréfutables, si du moins elles ne sont pas nécessaires en tant que conditions de possibilité de la régulation délibérative; c'est le cas de celles qui militent en faveur du droit à un minimum d'éducation, de bien-être et de sécurité, conditions toutes essentielles pour inspirer le respect. Enfin, d'autres raisons encore pourraient consister en considérations culturellement variables, portant sur les limites de la propriété privée ou la position d'une certaine religion dans la vie publique, ou le besoin d'homogénéité culturelle.

\section{Seconde question}

Comment des raisons populaires de ce genre pourraient-elles être intégrées dans la vie politique, et utilisées de manière à réguler le gouvernement? Les élections seront évidemment de toute première importance, à la fois pour éviter toute prise abusive de pouvoir par une dynastie ou une coterie quelconques, et pour fournir un contexte approprié au genre de discussion et de délibération publiques qui permettent aux raisons populaires de prendre corps et d'atteindre à la reconnaissance ${ }^{8}$. Mais il est tout aussi évident qu'il y aura des garde-fous et des institutions de nature non électorale qui aideront à s'assurer, en premier lieu, qu'aucune décision collective ne sera prise

7. Il est possible que John Rawls (I999) ait souvent en tête quelque chose comme les raisons populaires lorsqu'il parle des raisons publiques, et mes idées ont clairement été influencées par son propos. Je préfère cependant parler de raisons populaires, pour souligner certains points qui sont absents chez Rawls et qu'il aurait peut-être même rejetés: tout d'abord, le fait qu'elles soient le résultat du débat en cours; en second lieu, le fait qu'elles soient pertinentes pour un tel débat, quel que soit l'endroit, privé ou public, formel ou informel, dans lequel il a lieu; et en troisième lieu, le fait qu'en principe les raisons populaire qui opèrent au sein d'une société, ou même dans un espace public international, ne sont pas nécessairement des raisons qui véhiculent une force morale indépendante. Le langage des raisons populaires, tel que je l'utilise ici, est peut-être davantage dans l'esprit d'Habermas (I984, I989) que dans celui de Rawls. Je remercie Tim Scanlon pour notre discussion sur ce sujet.

8. Knights (2005) apporte une preuve convaincante du fait que c'est la fréquence des élections, à la fin du dix-septième siècle et au début du dix-huitième siècle, de même que l'existence d'une culture du pamphlet, des journaux et des clubs, qui ont permis la formation d'une politique discursive: une politique dans laquelle les différentes factions, avec leurs intérêts, ont donné lieu à des partis idéologiques qui se concentraient sur des différences de politique publique ouvertes à la négociation, de manière délibérative. 
en faveur d'options que les raisons populaires proscriraient; et, à s'assurer, en second lieu, que les décisions collectives par lesquelles on arbitrera entre d'autres options, plus ou moins acceptables, seront toujours prises sur la base de procédures que les raisons populaires reconnaîtront comme valides: selon le contexte, celles-ci pourront consister en un vote parlementaire, en un recours à une instance impartiale ou un ensemble d'experts, en un référendum général, et d'autres moyens.

Les instruments de nature non électorale auxquels on fera appel seront de nature variée, selon ce qu'exige l'implémentation de la régulation délibérative et les influences qu'exercent les raisons particulières validées par la discussion publique. Il est presque certain qu'elles incluront des contraintes sous la forme de lois, pesant sur la manière dont le gouvernement agit; des contraintes relatives aux droits individuels, pesant sur ce qu'il fait; des restrictions institutionnelles comme la séparation des pouvoirs, des mesures de responsabilisation et la dépolitisation de certaines décisions; et, ce qui est peut-être le plus important, le fait que les gens ordinaires et leurs représentants soient exposés à un pouvoir de surveillance et de contestation effectif. Les institutions électorales composeront avec ces éléments afin de créer une dynamique d'échange spécifiquement démocratique entre ceux qui sont dans le gouvernement, ceux qui se trouvent en dehors, et les gens ordinaires. Si cette dynamique fonctionne bien, alors elle permettra de s'assurer que le gouvernement n'agit pas contre les raisons populaires, et que, quand les raisons populaires exigent qu'il agisse, il agisse effectivement. Il respecte alors les exigences des raisons populaires, que ce soit dans la manière dont il agit, ou dans la manière dont il échoue à agir.

\section{Troisième question}

Le modèle du régime régulé de manière délibérative nous donne-t-il une représentation plausible du pouvoir populaire et nous permet-il de résoudre l'énigme démocratique? Je crois que c'est le cas. Il implique un système dans lequel les individus influencent le gouvernement dans différents lieux, comme on l'a vu, et à bien des niveaux. Si tout se passe à peu près bien, cette influence devrait avoir pour effet systématique — bien que peut-être seulement à long terme - de discipliner le gouvernement de sorte que les politiques ou les processus qui ne respectent pas les raisons populaires soient mis sur la touche. Et cet effet devrait être bien reçu de toute part, pour autant que l'on accepte en général que ces raisons sont celles qui devraient prévaloir dans la vie publique. Cette acceptation se manifestera dans la manière dont les individus s'attendront à ce que les autres, y compris au sein du gouvernement, se comportent lorsqu'ils défendront leurs causes.

Si les institutions électorales et non électorales qui prévalent en vertu de l'acceptation générale facilitent bien la régulation délibérative du gouvernement, alors on peut considérer que le réseau qu'elles constituent incarne bien le pouvoir du peuple. De nombreux acteurs joueront un rôle au sein de 
ce réseau, depuis les citoyens dans leurs rôles électoral et contestataire, jusqu'à ceux qu'ils éliront aux postes législatifs et exécutifs, en passant par ceux qui seront invités à jouer un rôle judiciaire, ou ceux qui travailleront dans les banques centrales ou les commissions électorales, et auxquelles les décisions publiques seront déléguées. Le réseau impliqué tâchera généralement de satisfaire un certain modèle global, plus ou moins cohérent, de résultats - sans quoi la société sombrerait dans l'anarchie — et, ce faisant, il lui faudra être fidèle aux types de raisons valorisées dans la discussion publique. Il devra ainsi exclure les politiques et procédés qui sont en contradiction avec ces considérations.

Nous sommes à présent en mesure de voir comment l'approche esquissée ici peut permettre de résoudre l'énigme démocratique, selon une stratégie réinterprétative parallèle à celle que l'on trouve chez Rousseau, bien qu'elles soient très différentes l'une de l'autre. La régulation délibérative constitue une forme de pouvoir populaire, en vertu de l'argument que l'on vient de proposer, et les raisons populaires qu'elle met en jeu laissent espérer que le régime ainsi imposé assurera la légitimité. Il est certain qu'il n'exclut pas le danger d'une tyrannie de la majorité. Nous pouvons alors réinterpréter les trois axiomes démocratiques d'une manière qui les rende individuellement convaincants, et cohérents les uns avec les autres.

- Axiome de l'équivalence: la démocratie signifie le pouvoir au peuple, régulé de manière délibérative.

- Axiome de la légitimité: la démocratie implique la légitimité.

- Axiome de la tyrannie: le pouvoir populaire n'implique pas en luimême la légitimité.

L'important, dans cette dernière section, n'était pas de fournir une défense convaincante de l'idée de démocratie régulée de manière délibérative, ni des moyens institutionnels par lesquels elle pourrait être établie. Je m'y intéresse ailleurs (Pettit, 20I2). Selon moi, il est important de disposer d'une conception de la démocratie qui résolve l'énigme démocratique, et mon seul objectif ici a été de montrer l'existence d'un candidat qui promet d'être capable de le faire: un candidat qui se trouve en réalité dans une famille de candidats, car la régulation délibérative peut donner lieu à des interprétations variées ${ }^{9}$. Il ne faut pas se représenter la démocratie en termes purement électoraux et majoritaristes - bien que la faveur de nombreux spécialistes (Przeworksi, I999) aille de ce côté-là —, du moins pas si on veut

9. Si on comprend les commentaires de Ronald Dworkin de manière non éclectique, comme on l'a souligné plus haut, on peut alors situer son approche dans cette famille. Et il en va de même pour l'approche adoptée par Jürgen Habermas $($ I996, 42) lorsqu'il soutient qu'un régime de droits individuels est nécessaire à la participation délibérative dans le gouvernement. Tout dépend de la manière dont on comprend l'expression "participation délibérative", notamment, si on conçoit cette catégorie de manière assez large pour qu'elle inclue ou recouvre celle de régulation délibérative. 
résoudre l'énigme démocratique. Et il n'est pas nécessaire de la penser ainsi. Une approche différente existe, bien qu'elle n'ait pas reçu jusqu'à présent toute l'attention qu'elle méritait, et dont elle aurait besoin ${ }^{10}$.

* Traduit de l'anglais par Aude Bandini

\section{Bibliographie}

Bohman, J. and W. Rehg, Eds. (I997). Deliberative Democracy: Essays on Reason and Politics. Cambridge, Mass., MIT Press.

Cohen, J. (1989). Deliberation and Democratic Legitimacy. The Good Polity. A. Hamlin and P. Pettit: I7-34.

Coleman, J. and J. Ferejohn (I986). "Democracy and Social Choice." Ethics 97 : 6-25.

Dworkin, R. (2006). Is Democracy Possible Here? Principles for a New Political Debate. Princeton, Princeton University Press.

Elster, J., Ed. (I998). Deliberative Democracy. Cambridge, Cambridge University Press.

Garland, D. (200I). The Culture of Control: Crime and Social Order in Contemporary Society. Chicago, University of Chicago Press.

Gutmann, A. and D. Thompson (I996). Democracy and Disagreement. Cambridge, Mass, Harvard University Press.

Habermas, J. (1984, I989). A Theory of Communicative Action, Vols I and 2. Cambridge, Polity Press.

Habermas, J. (I996). Postscript to Between Facts and Norms. Habermas, Modernity and Law. M. Deflem. London, Sage: 135-50.

Hobbes, T. (I994). Leviathan. ed E.Curley. Indianapolis, Hackett.

Holmes, S. (I995). Passions and Constraint: On the Theory of Liberal Democracy. Chicago, University of Chicago Press.

Knights, M. (2005). Representation and Misrepresentation in Later Stuart Britain: Partisanship and Political Culture. Oxford, Oxford University Press.

List, C. (2006). "The Discursive Dilemma and Public Reason.” Ethics I I 6: 362-402.

List, C. and P. Pettit (2002). "Aggregating Sets of Judgments: An Impossibility Result." Economics and Philosophy i 8: 89-1 Iо.

List, C. and P. Pettit (20II). Group Agency: The Possibility, Design and Status of Corporate Agents. Oxford, Oxford University Press.

Mayerfield, J. (2009). "Democratic Legitimacy of International Human Rights Law." Indiana International and Comparative Law Review I9.

Pettit, P. (200I). A Theory of Freedom: From the Psychology to the Politics of Agency. Cambridge and New York, Polity and Oxford University Press.

Pettit, P. (2003a). Deliberative Democcracy, the Discursive Dilemma, and Republican Theory. Philosophy, Politics and Society Vol 7: Debating Deliberative Democracy. J. Fishkin and P. Laslett. Cambridge, Cambridge University Press: I38-62.

10. Une version de cet article a été présentée au titre de Inaugural Edmund Burke Lecture, au Trinity College de Dublin, en 2007. J'ai tiré grand bénéfice des commentaires du public, et en particulier des remarques proposées par mon répondant, Leif Wenar. 
Pettit, P. (2003b). Groups with Minds of their Own. Socializing Metaphysics. F. Schmitt. New York, Rowan and Littlefield.

Pettit, P. (2007). "Responsibility Incorporated.” Ethics I I7.

Pettit, P. (2012). On the People's Terms: A Republican Theory and Model of Democracy. Cambridge, Cambridge University Press.

Przeworksi, A. (I999). A Minimalist Conception of Democracy: A Defense. Democracy's Value. C. Hacker-Cordon and I. Shapiro. Cambridge, Cambridge University Press.

Riker, W. (I982). Liberalism against Populism. San Francisco, W.H. Freeman and Co.

Rousseau, J.-J. (I973). The Social Contract and Discourses. London, J.M. Dent \& Sons Ltd.

Simmons, A. J. (1979). Moral Principles and Political Obligations. Princeton, Princeton University Press. 